Éléments pour une étude des représentations de la canne à sucre chez les Ankave-Anga (Papouasie Nouvelle-Guinée)

\title{
Pascale Bonnemère
}

\section{(2) OpenEdition}

Journals

Édition électronique

URL : http://journals.openedition.org/jso/1503

DOI : $10.4000 /$ jso. 1503

ISSN : $1760-7256$

Éditeur

Société des océanistes

Édition imprimée

Date de publication : 1 décembre 2002

Pagination : 181-185

ISSN : 0300-953x

\section{Référence électronique}

Pascale Bonnemère, «Éléments pour une étude des représentations de la canne à sucre chez les Ankave-Anga (Papouasie Nouvelle-Guinée) », Journal de la Société des Océanistes [En ligne], 114-115 | Année 2002, mis en ligne le 26 mai 2008, consulté le 22 mars 2021. URL : http:// journals.openedition.org/jso/1503; DOI : https://doi.org/10.4000/jso.1503

\section{(c) (†) $\Theta$}

Journal de la société des océanistes est mis à disposition selon les termes de la Licence Creative Commons Attribution - Pas d'Utilisation Commerciale - Pas de Modification 4.0 International. 


\section{Éléments pour une étude des représentations de la canne à sucre chez les Ankave-Anga (Papouasie Nouvelle-Guinée)}

par

Pascale BONNEMÈRE *

\section{RÉSUMÉ}

Dans l'ensemble des représentations qu'ont les Ankave des aliments, la canne à sucre est associée sans ambiguïté aucune au lait maternel, du moins à première vue. Car les modalités de consommation de celle-ci lors des rituels masculins révèlent une situation plus complexe, que l'auteur analyse à la fois dans le contexte ethnographique concerné et à l'aune de ce que l'on sait des associations entre les substances corporelles et certains aliments dans d'autres groupes anga.

MoTS-CLÉS : canne à sucre, maternité, initiations masculines, homosexualité ritualisée, comparatisme, transformations.

Venue parler d'ethnobotanique avec Jacques Barrau dans les locaux désuets du Museum d'histoire naturelle, j'évoquais les relations de contiguïté symbolique entre certaines substances corporelles et certains végétaux chez les Ankave-Anga lorsqu'il m'arrêta un instant pour grimper sur une chaise et attraper plusieurs tirésà-part stockés sur une étagère haut placée. L'un de ces articles, intitulé « Canna mellis : croquis historique et biogéographique de la canne à sucre », était illustré, outre de plusieurs planches, d'une photographie figurant un jeune homme kanak «buvant» une canne à sucre

\begin{abstract}
In the set of representations the Ankave have elaborated concerning food and nurturing, sugar cane seems unambiguously associated with maternal milk. Yet, the ways sugar cane is consumed during male initiations point to a more complex situation, which the author analyzes in this particular ethnographic context as well as with respect to what is known of the connections other Angans make between bodily substances and several foods.
\end{abstract}

KEYwORDS : sugar cane, motherhood, male initiations, ritualized homosexuality, comparatism, transformations.

(Barrau, 1988 : 165). Or, en visionnant récemment les films des initiations masculines organisées en 1994 par les Ankave, cette photographie et sa légende me sont revenues en mémoire.

C'est pourquoi je voudrais m'attacher ici à analyser les représentations locales du cultivar Saccharum officinarum L. dont le principal centre d'origine est la Nouvelle-Guinée ${ }^{1}$. Aux côtés du pandanus rouge et de quelques végétaux à la croissance spontanée, la canne à sucre et ses trente-cinq variétés nommées occupent une place de choix dans l'univers symbolique de la croissance des êtres humains de cette société.

1. D'après des informations plus récentes, la canne à sucre pourrait en fait être un hybride de Saccharum sinense de Chine et d'une espèce de Nouvelle-Guinée (Spriggs nd). 
Elle joue par exemple un rôle de premier plan lors des rituels masculins qui transforment, diton, les jeunes garçons en hommes physiquement matures et moralement adultes.

La canne à sucre (ngwen') appartient, comme les bananiers, à l'ensemble des " arbres au tronc rempli de liquide » (wi'we'). Dans leurs jardins, les Ankave en plantent les diverses variétés, distinguées selon la couleur (pourpre, jaune, vert) et le dessin (uni ou strié) de leur canne, au milieu des tubercules qui composent l'essentiel de leur alimentation (taros, Xanthosoma sagittifolium et patates douces, Ipomea batatas). Difficile à qualifier de nourriture - elle n'est jamais consommée au cours d'un repas par exemple —, la canne à sucre est avant tout une gourmandise que les enfants adorent et que les femmes emportent pour étancher leur soif lorsqu'elles partent travailler ensemble dans les jardins. Au bout de quelques heures, elles feront une pause et partageront des morceaux de canne en discutant les derniers potins. Aliment-plaisir donc, le jus de canne à sucre est consommé par tout le monde, sur une base quotidienne et sans restriction aucune. En outre, dans des circonstances où il existe un risque d'hémorragie, comme l'initiation, à cause de la perforation du septum des jeunes garçons, ou encore lors du premier accouchement d'une épouse ${ }^{2}$, il remplace l'eau de boisson car l'absorption de celle-ci favoriserait l'écoulement de sang. Aliment ni vraiment solide ni tout à fait liquide, le jus de canne à sucre a cette qualité tout à fait appréciable de désaltérer sans surcharger le corps de liquide.

Mais chez les Ankave, la principale caractéristique du jus de canne à sucre est d'entretenir un rapport de contiguité symbolique avec le lait maternel. Il est en effet l'unique aliment qu'une femme qui vient d'accoucher consomme car lui seul permet à la montée de lait d'avoir lieu, autrement dit au colostrum de s'épaissir et de se transformer en lait nourricier. De même, lors du sevrage (qui a lieu deux à trois années après la naissance), la première nourriture de substitution qu'une mère propose à un enfant réclamant le sein avec trop d'insistance est du jus de canne à sucre, qu'elle extrait elle-même par écrasement entre les dents et lui transmet de bouche à bouche. Enfin, si le lait maternel s'est tari, sans doute à la suite d'une rupture du tabou sur les relations sexuelles pendant l'allaitement, la seule solution pour le faire couler à nouveau est de boire le jus d'une canne à sucre.

Devant un tel système de représentations, on serait tenté de déduire que le jus de canne à sucre est associé à la maternité. Pourtant, l'analyse des modalités bien particulières selon lesquelles les spécialistes rituels donnent à consommer des cannes à sucre aux novices au cours des initiations masculines révèle une situation bien plus complexe.

Le cycle initiatique ankave comporte trois stades principaux : lorsqu'ils ont entre huit et douze ans, les garçons ont la cloison nasale percée ; quelques semaines à un an plus tard, ils sont frottés de graines de pandanus rouge ; enfin, lors de la naissance de leur premier enfant, les jeunes pères subissent un rite à l'issue duquel ils reçoivent un collier en rotin sur lequel sont fixées deux " défenses » de cochon et qui marque leur nouveau statut.

La première consommation rituelle de canne à sucre a lieu après que les novices aient absorbé une mixture faite de gingembre (Curcuma longa), de sel végétal ${ }^{3}$, de râpures de l'écorce très parfumée de Litsea toleiana et de graines cuites d'une Cucurbitacée à la peau rouge (Melothria sp.). On dit que cette puissante mixture, qui leur est enfournée violemment dans la bouche, " abîme » et "brûle » le foie et "assèche » la gorge. Le jus des cannes à sucre qu'ils boivent ensuite en atténue les effets en refroidissant leur corps. Or, les trois cultivars qui peuvent être donnés à cette occasion (xarena, imenenge' et sindexe') sont interdits aux hommes dont l'épouse est enceinte de leur premier enfant ainsi qu'aux femmes en temps ordinaire. La seule occasion où elles doivent les consommer est la veille du jour où l'on perfore le septum de leur fils ${ }^{4}$; mais, en tout état de cause, elles ne peuvent jamais les récolter.

$\mathrm{Si}$ l'on veut comprendre le système des représentations dont la canne à sucre fait l'objet, il faut donc se garder d'en considérer les différents cultivars comme équivalents les uns des autres mais bien plutôt analyser les contextes dans lesquels chacun d'entre eux intervient.

Les trois cultivars de canne à sucre mentionnés ci-dessus sont consommés en contexte rituel masculin et jamais - à une exception près, on l'a vu - par les femmes. En outre, les novices doi-

2. Notons ici qu'en vertu d'un système de représentations tout à fait particulier (Bonnemère, nd), c'est bien sur le mari d'une femme enceinte pour la première fois et non sur la femme elle-même que repose l'interdiction de consommer de l'eau pendant la grossesse.

3. Voir Lemonnier, 1984.

4. L'analyse de cette consommation particulière dépasse le cadre du présent article ; elle renverrait à coup sûr à la perception locale du rapport entre une mère et son garçon novice, que j'ai qualifiée ailleurs de « symbiotique » (Bonnemère, à paraître). 
vent sucer les courts segments de canne qu'on leur a donnés d'une manière inconnue dans les comportements alimentaires mais qui rappelle les fellations rituelles pratiquées chez les Anga du nord. Plutôt que de broyer la matière fibreuse de la canne après avoir retiré la peau qui l'enserre, les novices doivent en effet placer dans la bouche le segment qui leur a été remis sans avoir fait cette opération préalable "d'épluchage ». Ils en extraient alors le jus en l'aspirant après avoir écrasé la peau entre leurs dents. En outre, les débris sont ensuite méticuleusement récupérés par le frère aîné ou l'un des cousins de chaque garçon et déposés tous ensemble au pied d'un grand arbre au plus profond de la forêt, là où les femmes ne se rendent jamais. L'entreprise est délicate car il faut surveiller les cris d'oiseaux pendant toute sa durée. Les Ankave considèrent en effet que le chant de certains oiseaux est un signe de bon augure alors que d'autres annoncent des catastrophes. Selon le cri entendu lors de la récupération et du dépôt des restes de canne à sucre, le garçon et ses parents auront une longue vie, exempte de maladie, ou seront au contraire confrontés à des épreuves douloureuses.

Les modalités spécifiques de la consommation de ces cultivars de canne à sucre particuliers et du traitement de leurs débris attestent du caractère éminemment rituel de ces opérations. Non seulement leur consommation est quasi-exclusivement réservée aux hommes, mais celle-ci se distingue aussi de la façon habituelle de manger les autres cultivars qui sont, eux, associés à la féminité et considérés comme des substituts du lait maternel. En outre, les morceaux de peau non consommés par les jeunes garçons sont placés dans un endroit caché des femmes.

Enfin, lors de cette consommation masculine de jus de canne à sucre, sont dispensés des leçons sur les obligations de partage et des messages sur les comportements à adopter en diverses circonstances, notamment avec les femmes. Il est également demandé aux novices d'être très attentifs aux cris des oiseaux lorsqu'ils mangent, car les noms de certains d'entre eux (par exemple abaxe terange et xoxapi) évoquent le mot "femme» (abaxe) ou des insultes à caractère sexuel (api renvoyant ici à ipia, «sexe de femme»), tout comme les cris ou sifflements de quelques autres. Si l'un de ces oiseaux chante, les garçons doivent aussitôt cracher la nourriture qu'ils ont dans la bouche. Cette pratique souligne l'incompatibilité entre cette consommation rituelle et tout ce qui a trait à la féminité ou évoque les femmes, ce qui confirmerait que ces trois cultivars de canne à sucre particuliers n'ont pas grand chose de commun avec ceux qui stimulent la production de lait dans le corps de celles-ci.

Tous ces faits tendent à valider l'hypothèse selon laquelle la majorité des cultivars de canne à sucre entretiennent bien une relation de contiguité symbolique avec le lait maternel mais trois d'entre eux (xarena, imenenge' et sindexe') n'en sont pas moins associés à la masculinité. L'analyse de l'interdit de consommer l'une ou l'autre de ces trois cannes qui pèse sur le futur père d'un premier enfant permet même de proposer l'hypothèse que ces cultivars sont en fait considérés comme des substituts du sperme. Il est en effet possible de rapprocher cette prohibition du tabou portant sur les relations sexuelles pendant la grossesse. Tout comme le sperme est néfaste au développement du fœtus, dans ce genre de systèmes de représentations où un aliment consommé par une personne peut avoir un effet sur le corps d'une autre qui lui est reliée (Bamford, 1998 ; Bonnemère, nd), le jus de ces cannes à sucre est prohibé pour un futur père car, s'il en consommait, il entraverait la croissance intra-utérine de son enfant à naître.

Le mode spécial de consommation de ces trois cultivars de canne à sucre évoque la description que donne Herdt du « rite des flûtes » (« the flute ceremony », Herdt, 1987 : 145-155) organisée par les Sambia au troisième jour des cérémonies du premier stade des initiations (idem: 128). L'analyse qu'il fait de ces instruments comme support de la préparation à la fellation (idem : 148) permet alors de rapprocher, par transitivité, le rite de la canne à sucre des Ankave aux pratiques homosexuelles ritualisées de deux groupes anga du nord, les Baruya et les Sambia (Godelier, 1982; Herdt, 1987).

On pourrait donc partir de l'hypothèse que l'on se trouve devant un système de transformations à l'échelle de l'ensemble des groupes anga, dans lequel les significations données au jus de canne à sucre ainsi que les modalités de sa consommation varieraient ensemble. Cependant, si le mode de consommation des cannes à sucre lors des initiations ankave est comparable aux pratiques homosexuelles ritualisées des Sambia, induisant alors une association possible entre le jus sucré et le sperme, il n'est pas pour autant légitime d'en inférer que les deux groupes font jouer à ces substances le même rôle. La transformation se situe ici dans un double registre : celui de l'élément lui-même, corporel ou végétal, et celui de sa fonction ${ }^{5}$ car, si les jeunes novices sambia grandissent grâce au sperme de

5. Fonction que l'on analyse, on l'a vu, en prenant essentiellement en compte les modalités techniques de sa consommation et le moment où il entre en action dans la séquence rituelle (voir ci-dessous). 
leurs aînés célibataires, on serait bien en peine, en l'absence de commentaires par les spécialistes rituels, d'interpréter le sens de la consommation rituelle du jus de canne à sucre chez les Ankave.

Chez ces derniers, la consommation de jus de canne à sucre a lieu bien plus tôt que chez les Sambia dans la séquence des actes rituels, et notamment avant leur renaissance symbolique matérialisée par la progression difficile des novices et de leurs parrains dans un corridor de branchages étroit (Bonnemère, 2001 : 23, 25-26). Les Ankave font précéder cette renaissance d'une ingestion secrète de la sauce extraite des fruits cuits du pandanus rouge, qui reproduit quant à elle le processus de la gestation. Une telle interprétation se fonde, d'une part, sur le discours des mères des garçons, d'autre part, sur la congruence des interdits alimentaires dans les deux contextes de la grossesse et de l'initiation, et sur la similitude des comportements requis des novices reclus en forêt et des mères rassemblées dans un grand abri au village (Bonnemère, 1996 : 348).

Compte tenu de la position différentielle de la consommation du jus de canne à sucre ankave et des rites de fellation sambia dans les séquences de leur cycle cérémoniel respectif, on ne peut logiquement interpréter ces deux actions rituelles comme des équivalents. Les pratiques homosexuelles ritualisées de ce groupe anga du nord ont été interprétées comme une forme d'allaitement symbolique (Herdt, 1981 : 234 ; Bonnemère, $2001: 26$ ) qui prend place, dans l'ordre des actes rituels, après le passage dans le corridor de branchages dont l'aspect « ensanglanté » ${ }^{6}$ a permis de confirmer qu'il s'agissait bien là d'une re-naissance.

Ainsi, le rapport qui existerait entre jus de canne à sucre et sperme chez les Anga est complexe et a subi des distorsions et des ajustements de divers ordres. En tout état de cause, il ne constitue qu'un exemple au sein d'un ensemble de transformations qui forment probablement système. Par exemple, le rôle nourricier que les Anga du nord font jouer au sperme est rempli, dans les représentations de la procréation des Ankave, par le sang maternel et par son substitut végétal, le jus du pandanus rouge. Ce qui a pour effet de valoriser le rôle attribué à la mère dans ce processus, qui est pour eux à l'origine de la crois- sance du fœtus, alors que, chez les premiers, celle-ci est réduite à n'être qu'un simple contenant (Herdt 1981 : 193). La permutation qui voit la place occupée par le sperme chez les uns, investie par le sang chez les autres, retentit directement sur les représentations associées à chaque sexe et à leur rôle respectif dans la reproduction des êtres humains. À leur tour, et comme on l'a montré ailleurs, ces représentations ne sont pas sans conséquence sur les aspects sociaux des relations entre les hommes et les femmes (Bonnemère, $1996:$ ch.10).

Enfin, tandis que, pour les Baruya, ce sont le soleil et la lune qui ont donné aux hommes les objets sacrés (Godelier, 1982: 136), dans les mythes ankave, les plantes et les minéraux utilisés dans les initiations sont apparus à l'endroit où le sang d'un homme primordial assassiné s'est écoulé. On se trouve donc ici en face d'un ensemble de transformations qui se renvoient les unes aux autres (Lemonnier, nd).

Dans la mesure où les groupes anga actuels sont le résultat d'un long processus de différenciation culturelle amorcé il y a probablement plusieurs millénaires à partir d'une même population de la région de Menyamya (Lemonnier, 1997), il n'est pas déraisonnable d'imaginer que les systèmes de représentation et les pratiques de ces groupes ont subi de telles transformations. Pour s'en tenir au propos de ce court article, les représentations attachées aux trois cultivars de canne à sucre que les Ankave ont isolés de tous les autres pourraient témoigner d'une pratique ancienne, l'homosexualité ritualisée, depuis longtemps disparue chez eux mais que d'autres ont conservée jusqu'à une époque récente.

Enfin, l'exemple des représentations de la canne à sucre chez les Ankave permet, me semble-t-il, de réfléchir au devoir de prudence et d'attention extrême aux détails qui repose sur l'ethnologue, tout particulièrement lorsqu'il analyse les idées qu'ont les membres d'une société à propos d'un élément de leur environnement. Considérer la canne à sucre, ou toute autre plante, comme une entité sans tenir compte du fait que ses différents cultivars puissent être conçus comme indépendants les uns des autres ${ }^{7}$ des associations qui leur sont propres, serait en effet passer à côté de la richesse d'un univers symbolique particulier.

6. «Pieces of the shaman's red headband are tied up in branches of the green mass [...]. The effect is calculated to be bizarre. The initiates are pushed into this 'bloody' chaos, tied to the back of their rituel sponsors, so they cannot escape » (Herdt, 1987 : 141).

7. Il en va évidemment de même pour certains animaux : chez les Ankave par exemple, tous les marsupiaux ou tous les oiseaux ne sont pas considérés comme dotés des mêmes attributs et caractéristiques et chaque variété fait l'objet de représentations particulières. 


\section{RÉFÉRENCES}

BAMFORD, Sandra C., 1998. To eat for another : taboo and the elicitation of bodily form among the Kamea of Papua New Guinea, in M. Lambek and A. Strathern (eds), Bodies and persons. Comparative perspectives from Africa and Melanesia, Cambridge : Cambridge University Press, pp. 158-171.

BARraU, Jacques, 1988. Canna mellis : croquis historique et biogéographique de la canne à sucre, Saccharum officinarum L. Graminées-Andropogonées, Journal d'Agriculture Traditionnelle et de Botanique Appliquée XXXV, pp. 159-173.

BONNEMÈRE, Pascale, 1996. Le pandanus rouge. Corps, différence des sexes et parenté chez les Ankave-Anga (Papouasie Nouvelle-Guinée), Paris: CNRS Éditions/Éditions de la MSH.

_, 2001. Two Forms of Masculine Ritualized Rebirth : The Melanesian Body and the Amazonian Cosmos, in T. Gregor and D. Tuzin (eds), Gender in Amazonia and Melanesia: An Exploration of the Comparative Method, Berkeley : University of California Press, pp. 17-44.

- , à paraître. When women enter the picture : Looking at Anga initiations from the mothers' angle, in P. Bonnemère (ed), The Unseen Characters. Women in Male Rituals of Papua New Guinea.
—, nd. «Faire pour autrui : la construction relationnelle de l'individu chez les Ankave-Anga (Papouasie Nouvelle-Guinée) », texte manuscrit (séminaire de DEA de l'Université de Provence, 15 janvier 1999).

GODELIER, Maurice, 1982. La production des Grands Hommes, Paris : Fayard.

Herdt, Gilbert H., 1981. Guardians of the Flutes. Idioms of Masculinity, Chicago and London: The University of Chicago Press.

_, 1987. The Sambia. Ritual and Gender in New Guinea, New York : Holt, Rinehart and Winston.

LEMONNIER, Pierre, 1984. La production de sel végétal chez les Anga (Papouasie Nouvelle-Guinée), Journal d'Agriculture Traditionnelle et de Botanique Appliquée XXXI (1-2), pp. 71-126.

_, 1997. 'Mipela wan bilas'. Identité et variabilité socioculturelle chez les Anga de Papouasie Nouvelle-Guinée, in S. Tcherkézoff et F. DouaireMarsaudon (eds), Le Pacifique sud aujourd'hui. Identités et transformations culturelles, Paris : Éditions du CNRS, pp. 197-227.

—, nd. « La mémoire de l'os ou les vacances de monsieur Soleil », texte manuscrit (séminaire de M. Godelier, EHESS - Paris, 24 janvier 2000).

SPRIGGs, Matthew, nd. «Pacific Archaeology : What's new ? », papier présenté au séminaire du CREDO, 23 avril 2002. 